\title{
Estágios curriculares no SUS: experiências da Faculdade de Odontologia da UFRGS
}

Cristine Maria Warmling*, Eloá Rossoni*, Fernando Neves Hugo**, Ramona Fernanda Ceriotti Toassi***, Vânia Aita de Lemos****, Sonia Maria Blauth de Slavutzki*****, Solange Bercht*****, Ângela Antunes Nunes******, Arisson Rocha da Rosa*******

\author{
* Doutora em Educação, Docente FO/UFRGS \\ ** Doutor em Saúde Coletiva, Docente FO/UFRGS \\ *** Doutora em Educação, Docente FO/UFRGS \\ ***** Mestre em Odontologia, Docente Licenciada FO/UFRGS \\ ****** Doutora em Odontologia, Docente FO/UFRGS \\ ***** Doutora em Saúde Coletiva, Docente FO/UFRGS \\ ******* Especialista em Odontologia em Saúde Coletiva, Docente FO/UFRGS
}

\section{RESUMO}

Um dos objetivos principais das propostas de mudança de paradigma no ensino odontológico do país tem sido o de conformar o perfil profissional do cirurgião-dentista de modo a torná-lo mais ajustado às exigências ditadas pelo Sistema Único de Saúde. Mas esse é um modelo de formação incipiente e que ainda carece de consensos em torno do modo como formar esses profissionais. O objetivo deste artigo é descrever e avaliar processos pedagógicos, técnicos e políticos produzidos no percurso de implantação dos estágios curriculares de odontologia da Faculdade de Odontologia da Universidade Federal do Rio Grande do Sul, apontando aspectos envolvidos nas experiências de reorientação da formação do cirurgião-dentista para sua atuação no Sistema Único de Saúde. Foram analisadas produções escritas e depoimentos de docentes, documentos e relatórios institucionais e pesquisas escolares. As redes de atenção e ensino em saúde bucal encontram-se em processo de estruturação, desafios precisam ser superados: expansão limitada da atenção primária à saúde e, consequentemente, dos campos de estágios; necessidade de avanços nas discussões sobre o papel, atribuições e institucionalizações do preceptor/trabalhador e do tutor/docente; as incompreensões, ainda persistentes, a respeito dos estágios, tanto na instituição de ensino superior como na gestão e nos serviços do SUS; questões de financiamento; discurso hegemônico da clínica liberal-privatista e seus reflexos no embate constante entre tutores/preceptores e discentes; limites impostos pelo desenho fragmentado da rede de atenção em saúde.

\section{DESCRITORES}

Ensino. Educação. Estágio. Atenção primária à saúde. Saúde bucal. Extensão comunitária.

$\Delta$ III Conferência Nacional de Saúde Bucal (III CNSB), em 2004, apresentou o seguinte diagnóstico sobre a formação de trabalhadores para a área da odontologia:

- há dissociação entre ensino e realidade brasileira, e também,

- falta de debate social sobre características políticas e técnicas dos profissionais formados.

A superação desses problemas, na opinião dos conferencistas, passa por redefinir o modelo de formação, integrar docência serviço e pesquisa, estimular parcerias entre Instituições de Ensino Superior (IES) e Secretarias Estaduais (SES) e Municipais de Saúde (SMS), estabelecer novos mecanismos de avaliação dos cursos e realizar capacitação dos profissionais de saúde. ${ }^{6}$

Um dos objetivos principais das propostas de mudança de paradigma no ensino odontológico do país tem sido o de conformar o perfil profissional do ci- 
rurgião-dentista de modo a torná-lo mais ajustado às exigências ditadas pelo Sistema Único de Saúde (SUS). ${ }^{4}$ As novas experiências curriculares dos cursos de odontologia devem proporcionar aos acadêmicos atuar nos serviços de saúde do SUS. Para isso, nos municípios e também nos estados as redes de instituições de ensino e de serviço se integram através da operacionalização de ações de integração ensinoserviço-comunidade. ${ }^{29}$

A experiência vivenciada pela Faculdade de Odontologia da Universidade Federal do Rio Grande do Sul (FO/UFRGS) é um exemplo a ser estudado quando se quer compreender de que modo essas transformações tem ocorrido. Essa instituição pública tem pautado seu projeto político pedagógico baseando-se no panorama político nacional. Procurou reorganizar seu currículo para, dentre outros objetivos, integrar atividades acadêmicas com o mundo do trabalho no SUS. Os estágios curriculares supervisionados recebem papel de destaque neste sentido. Nas novas proposições curriculares houve aumento substancial de carga horária para a realização dos estágios nos SUS. Desde o ano de 2006, os estágios têm sido implantados de forma progressiva, buscando propiciar aos estudantes do curso a inserção nos serviços de Atenção Primária à Saúde (APS) através da atuação em Unidades Básicas de Saúde (UBS) e de Estratégia de Saúde da Família (USF/ESF), assim como, em serviços de Gestão e Atenção Especializada na Saúde Bucal. ${ }^{1,15}$

A implantação das mudanças curriculares da FO/ UFRGS, que ocorre desde 2005, e especialmente dos estágios curriculares, tem sido objeto de debate e avaliação em diferentes momentos institucionais, incluindo pesquisas. Resultam desse processo documentos e relatórios. Recentemente, por exemplo, se realizou uma oficina de avaliação curricular com a participação de gestores, usuários, controle social, preceptores, tutores, docentes e discentes. Durante a oficina os inúmeros desafios cotidianos que acompanham as mudanças curriculares e a realização dos estágios curriculares foram discutidos, analisados e relatados. ${ }^{26}$

Nesse contexto, o objetivo deste artigo é descrever e avaliar processos pedagógicos, técnicos e políticos produzidos no percurso de implantação dos estágios curriculares de odontologia da FO/UFRGS, apontando aspectos envolvidos nas experiências de reorientação da formação do cirurgião-dentista para sua atuação no SUS. Para isso foram analisadas produções escritas e depoimentos de docentes, docu- mentos, relatórios institucionais e resultados de pesquisas acadêmicas.

\section{ESTÁGIOS CURRICULARES NO SUS: INTERMEDIAÇÃO ENTRE MUNDO DO TRABALHO E MUNDO ACADÊMICO}

Os novos percursos curriculares da graduação em odontologia devem dar conta de formar perfis profissionais predominantemente generalistas, mas esse é um modelo de formação incipiente e que ainda carece de consensos em torno do modo como formar esses profissionais. Em sua trajetória histórica desde a sua emergência enquanto ensino autônomo da medicina, inúmeras reformulações curriculares tem sido realizadas com divergentes objetivos de acordo com os contextos sociais. ${ }^{18,28}$ Para se construir, portanto, referenciais teóricos que possibilitem análises das mudanças na formação do cirurgião-dentista na atualidade, é preciso, antes de tudo, perguntar-se, lembra Bercht (2008): Qual a natureza da sociedade atual? $\mathrm{E}$, como é que o trabalho do cirurgião-dentista deve se estabelecer em relação a ela?

Há uma nítida crise no mundo do trabalho, a palavra crise entendida aqui, como uma séria mudança que se manifesta em inúmeros aspectos:

- o desemprego estrutural,

- o trabalho morto (feito por máquinas),

- a contratação temporária e

- a precarização das condições de trabalho,

Enfim, a perda de garantias, ou seja, do próprio "direito" ao trabalho como uma forma de inserção social.

A crise do trabalho, da morfologia do trabalho, é também a crise da sociedade e do capitalismo contemporâneos, mostrando suas interfaces de insuficiências e inadequações. ${ }^{1}$

As mudanças mundiais do trabalho ocorridas no último século - sociais, políticas, econômicas, culturais e tecnológicas - afetam o cotidiano da prática odontológica e produzem desafios para a educação nesse campo. Diante da inadequação dos saberes fragmentados, frente a realidades de trabalho cada vez mais complexas e globais, emerge a necessidade de maior articulação entre conhecimentos e práticas. A hiperespecialização impede de ver o global, pois o fragmenta em parcelas. Segundo Montaigne, mais vale uma cabeça bem-feita que bem cheia. Cabeça bem-feita exige aptidão para colocar e tratar problemas, capacidade de ligar os saberes e lhes dar sentido. Reformar o ensino é reformar pensamentos. ${ }^{16}$ 
A questão que se impõe para a odontologia é, então, como produzir cabeças bem feitas? Quais as competências e habilidades necessárias ao novo perfil profissional do cirurgião-dentista generalista, e principalmente, como desenvolvê-las nos cursos de graduação em odontologia? O estágio pode constituir-se em um lócus privilegiado de aprendizado e de transformação na interface trabalho e mundo do saber?

O texto constitucional da Resolução CNE/CES N. 3 , de 19 de fevereiro de 2002, contém a política curricular atual para os cursos de graduação em odontologia. Estabelece que a formação do cirurgião-dentista deve contemplar o sistema de saúde vigente no país, a atenção integral da saúde num sistema regionalizado e hierarquizado de referência e contra-referência e o trabalho em equipe. ${ }^{4}$

Conectado com as políticas no campo da educação e da saúde o projeto político-pedagógico da FO/ UFRGS identificou a necessidade de constituição de novos paradigmas de ensino-aprendizagem para compor os diferentes momentos do percurso de formação dos alunos. Orientou a formação de um perfil profissiográfico mais ajustado ao que propõem as novas diretrizes curriculares nacionais para os cursos de graduação em odontologia. ${ }^{20}$

No percurso da operacionalização da aproximação entre o ensino de graduação na área da saúde e o SUS destacam-se dois programas nacionais:

- Programa Nacional de Reorientação da Formação Profissional em Saúde (Pró-Saúde) e

- Programa de Educação pelo Trabalho para a Saúde (Pet-Saúde) ${ }^{8,9}$

Esses programas têm por finalidade estimular os processos de reformulação dos cursos da área da saúde enfatizando a inserção das ações de formação desses cursos nos serviços do SUS. Expressam uma operacionalização dos princípios contidos nas políticas mais gerais do ensino e da saúde. A FO/UFRGS obteve financiamento para o desenvolvimento desses dois projetos que estimulam a integração ensinoserviço-comunidade no curso.

No resumo do projeto do Pró-saúde da FO/UFRGS está a intenção de direcionar a reformulação curricular para a aproximação da formação que se desenvolve na faculdade de odontologia com o SUS. O Pró-Saúde da FO/UFRGS foi estruturado a partir de um diagnóstico da inserção da Faculdade no SUS, tanto na perspectiva histórica, como a partir do novo projeto político pedagógico. Uma nova matriz curri- cular foi estabelecida atendendo as Diretrizes Curriculares para os cursos de Odontologia. Tanto os objetivos do curso como a sua estrutura didático-pedagógica voltaram-se para aproximar a formação profissional dos serviços. ${ }^{23}$

As duas Cartas Acordo estabelecidas entre a Fundação de Apoio da Universidade Federal do Rio Grande do Sul (FAURGS) e a Organização Pan-Americana da Saúde/Organização Mundial da Saúde (OPAS/ OMS) estabelecem os termos de aproximação entre a Faculdade de Odontologia e o SUS, definindo três eixos de orientação.

- Teórico: promover um Núcleo de Educação Permanente, consolidar um conceito de processo saúde-doença, resgatar o Centro de Pesquisa em Odontologia Social, criar banco de dados de base populacional, realizar avaliação sistemática, ampliar e diversificar a pós-graduação em direção ao SUS, constituir-se em ferramenta de disseminação de conhecimento.

- Prático: proporcionar condições de contextualização do SUS e capacidade crítica para mudanças, integrar-se aos serviços do SUS em todos os níveis de complexidade, oportunizar aprendizado no método clínico e de gestão.

- Pedagógico: organizar espaço multiprofissional sobre atenção primária, ter a Estratégia Saúde da Família como referencial prático, capacitar corpo docente, organizar espaços de encontro e de formação ético-político-pedagógica aos docentes e profissionais de saúde envolvidos no curso. ${ }^{21,22}$

De acordo com a nova matriz curricular o envolvimento com a rede de serviços deve ocorrer já no primeiro semestre do curso, este comprometimento é gradativo, sendo que o maior número de créditos planejados se dá nos dois últimos semestres quando os estágios forem desenvolvidos como principal atividade formativa discente. ${ }^{23}$

O propósito dos estágios curriculares da FO/UFRGS é constituir experiências de educação no trabalho através das quais os estudantes compreendam as conformações das redes de atenção à saúde que compõem o SUS. ${ }^{19}$ Para cumprir este objetivo os estágios são realizados no último ano do curso de odontologia (nono e décimo semestre) possuindo 465 horas de duração cada um deles. Em cinco turnos da semana os estudantes dispersam-se nas unidades de saúde e em serviços de atenção especializada do SUS municipal, sempre acompanhados por um preceptor cirurgião-dentista trabalhador. Em um turno semanal 
concentram-se na FO/UFRGS quando sob a orientação de docentes/tutores realizam atividades curriculares programadas tais como:

- debates temáticos com palestrantes convidados e docentes;

- apresentação de seminários;

- discussão de projetos terapêuticos singulares;

- relatos e discussão de situações vivenciadas;

- oficinas integração ensino-serviço;

- apresentação e discussão de relatórios do estágio.

Há ainda o turno dedicado a tutoria quando grupos menores focam-se em refletir e discutir com os seus respectivos tutores situações da realidade em que estão inseridos. ${ }^{24,27}$

Quando a academia se aproxima do mundo do trabalho para ensinar saúde pública, deve fazê-lo de forma construtiva, para auxiliar na busca de soluções cientificamente viáveis e eticamente comprometidas com o humano. O ensinar no SUS não pode ser reduzido ou entendido como uma tentativa de sanar as precariedades do sistema, quanto a recursos humanos, por exemplo, ou, por que "lá não tem ninguém para atender as pessoas".

\section{EDUCAÇÃo PERMANENTE E A FORMAÇÃo DO CIRURGIÃO-DENTISTA}

Esse contexto de transformação e aproximação do ensino ao SUS trouxe consigo, portanto, a necessidade de construir processos de discussão que visem ampliar a compreensão dos serviços sobre o ensino e vice versa. A educação permanente é um processo construído pelo setor saúde para efetuar essas relações orgânicas entre o ensino e as ações e serviços e entre docência e a atenção à saúde, com o intuito de superar programas de capacitações e atualizações para os trabalhadores da saúde. O desafio da educação permanente está em reduzir a distância entre escolas, serviços e comunidades. ${ }^{1,12,13}$

Percebendo a responsabilidade da instituição de ensino na educação permanente dos trabalhadores/ preceptores que atuam nos estágios, a FO/UFRGS tem desenvolvido projetos com este objetivo. Um exemplo foi uma ação de extensão universitária realizada em 2010 com o objetivo de oportunizar ainda mais a integração entre a FO/UFRGS e o SUS municipal. Os trabalhos se desenvolveram através de oficinas e debates em que participaram trabalhadores de nível superior, médio e fundamental da atenção básica, usuários, líderes comunitários, estudantes da graduação e da pós-graduação e docentes. Os objeti- vos e a dinâmica de funcionamento do estágio estiveram articulados com a ação de extensão em educação permanente realizada. ${ }^{29}$

Os cenários de aprendizado no SUS problematizados nas oficinas e debates da FO/UFRGS foram as redes de atenção à saúde do município de Porto Alegre. Organizou-se um cronograma para os encontros e a escolha das temáticas abordadas foi realizada pelo grupo de docentes condutores da ação priorizando-se pautas da PNSB:

- SUS,

- políticas de saúde bucal,

- resiliência e trabalho com famílias,

- vigilância e educação na saúde bucal,

- participação comunitária e controle social.

Estes temas serviram como mote para a problematização das práticas de atenção primária à saúde. Conduzindo os debates estavam gestores da saúde bucal das três esferas governamentais, docentes e trabalhadores com experiência nas temáticas discutidas e representantes do conselho municipal de saúde. ${ }^{29}$

Com o intuito de avaliar essa ação foi enviado via correio eletrônico aos preceptores a seguinte pergunta: "Como as oficinas realizadas contribuíram com os serviços e preceptoria?” A análise temática das respostas dos preceptores apontou que as atividades da educação permanente serviram para:

- trocar informações e experiências,

- fortificar conhecimentos,

- refletir sobre a realidade,

- promover ideias para projetos,

- partilhar experiências,

- saber como os outros serviços executam as normas editadas pela SMS,

- integrar a academia e o serviço,

- aproximar e valorizar os profissionais da rede,

- ampliar nossos conhecimentos,

- conectar mais efetivamente a teoria e a realidade dos serviços e espaço de reflexão. ${ }^{25}$

A ação de educação permanente foi realizada por meio de problematizações do cotidiano dos serviços de saúde e permitiu a construção e reconstrução reflexiva das práticas em saúde. Os resultados das discussões propiciaram uma visão de como está ocorrendo a formação no serviço, suas potencialidades e desafios de transformação da realidade e ofereceram subsídios para se refletir sobre estratégias técnicas, pedagógicas e políticas mais ajustadas. ${ }^{25}$ 


\section{MAQUINARIAS DE PRODUÇÃO DE AUTORIA: DIÁRIOS DE CAMPO}

Os desafios que se impõem ao ensino no trabalho poderiam ser apresentados como apenas teóricometodológicos, porém, tornam-se éticos na medida em que os espaços de trabalho/ensino envolvem elementos que servem de instrumento para as escolhas morais e éticas dos sujeitos.

Nesta perspectiva, o uso dos diários de campo permite um acompanhamento processual do envolvimento do aluno com seu campo de estágio. É espaço de registro de realidade, de descrição de relações interpessoais no mundo do trabalho, de interpretação de acontecimentos e enfrentamentos a partir da análise do referencial teórico dado em aula e, finalmente, de reflexão e expressão subjetivas. Sistematicamente se cria outras ideias, compartilhadas e discutidas com o grupo de colegas, tutores e preceptores. Essa busca expande o espaço do trabalho tornando o diário de campo obra particular do aluno. Esse jogo de constituição de autoria permite também enfrentar possibilidades e dificuldades do percurso ético, teórico e metodológico travado pelo aluno. É a tentativa de uma subjetivação diferente, muito necessária no ensino no trabalho.

Os diários de campo, territórios de vivências tanto do discente quanto do docente, proporcionam a confecção de cartografias que criam possibilidades de novos olhares e análises. Em conjunto com outras atividades desenvolvidas nos estágios visam uma apropriação do eu em relação com os outros e com o mundo propiciando oportunidades de construção de racionalidades éticas.

A autonomia é entendida como certa medida de possibilidades de ação que permitam escolhas e responsabilidades, como algo que se assume gradativamente em virtude da apropriação de sua parte nos acontecimentos e ligado intrinsecamente à noção que se adquire de si e de suas possibilidades de escolha.

Trabalhar com a confecção de diários de campo não é fácil, pois quanto mais esta técnica pedagógica é bem sucedida mais autoria possui o aluno exigindo um processo intenso de discussão com o professor e com o grupo. A quantidade de material elencado e a qualidade de sua análise aumenta gradativamente, gerando uma riqueza de conteúdos que pode dificultar a apreciação, exigindo tempo e concentração na análise das obras, cabendo reconhecer que o sucesso está ligado ao fato do crescimento do aluno escapar às possibilidades de sua avaliação. $\mathrm{O}$ que extravasa ao controle e a condução, enfim, a possibilidade de ir além do exigido e de surpreender é que possibilitam aos alunos a autonomia necessária à gestão do seu processo de aprendizado e a negociação com o processo de aprendizado dos seus colegas. A reflexão ética com esta proposta teórico-metodológica compõe um cenário onde são permitidos que os elementos identidários e de alteridade se encontrem e negociem entre si.

A tensão entre os mundos do ensino e do trabalho passa a constituir um foco de análise. Antes, o diário íntimo não passava de algo relativo à personalidade, agora, as relações da posição do pesquisador-estagiário passam a ser constituídas na interação vida-ciência.

\section{PROJETOS TERAPÊUTICOS SINGULARES: CULTIVO dO CUIDADO CLÍNICO.}

As mudanças curriculares que vem sendo realizadas nos cursos de formação dos cirurgiões-dentistas brasileiros nos últimos anos procuram imprimir renovação à prática odontológica. Essa produção de novas práticas cuidadoras em saúde bucal exige o agenciamento de novos saberes e métodos. ${ }^{17}$

Campos (2008) destaca a necessidade de se reformarem saberes e práticas com o intuito de aproximar a clínica da saúde coletiva. Esta ampliação da clínica deve ser entendida enquanto transformação da atenção individual e coletiva, de forma que se possibilite que outros aspectos do sujeito, e não apenas o biológico, possam ser compreendidos e trabalhados pelos profissionais. Os objetivos da clínica ampliada visam à produção de saúde e o aumento do grau de autonomia do usuário, família e da comunidade. ${ }^{11}$

Os estágios motivam integrações entre os campos da clínica e da saúde coletiva. Assim, na educação no trabalho, como na vida, integram-se conhecimentos técnicos ou clínicos e uma gama de temas e discussões que se referem ao campo da saúde coletiva, tal como no conceito de clínica ampliada.

Compreende-se clínica ampliada como uma clínica humanizada e compromissada, que não se limita ao que ocorre no consultório, pois considera aspectos sociais e culturais. Usa critérios mais subjetivos e éticos, pratica a escuta e a integração, levando em conta angústias, aflições, medos, preocupações e anseios dos pacientes. A contemporaneidade tem cobrado uma maior humanização das práticas odontológicas e os estágios trabalham nesta perspectiva ao desenvolverem o conceito de clínica ampliada com os alunos.

Junto a este contexto emerge a necessidade do uso e criação de novos processos de ensino-aprendizagem que possam dar conta dos desafios das trans- 
formações curriculares hoje vivenciadas nos cursos de odontologia. A possibilidade de construção de projetos terapêuticos singulares, por exemplo, assume um papel importante neste sentido. O objetivo é permitir a sistematização de experiências entrelaçando histórias de situações de saúde e doença bucal vivenciadas por comunidades, famílias e / ou indivíduos e diferentes possibilidades de gestão da clínica odontológica. As histórias se desenvolvem de maneira a permitir que os discentes possam exercitar o enfretamento de situações de saúde e doença bucal compreendendo a interação entre questões sociais, políticas e culturais mais abrangentes com o planejamento e execução de projetos terapêuticos singulares em saúde bucal inseridos nas redes de atenção à saúde.

Uma clínica que se torna ampliada, portanto, também a partir da incorporação dos sujeitos do cuidado (e de suas singularidades) como atores/ agentes das propostas terapêuticas construídas. Os estágios, mesmo quando se dispõe sobre atenção especializada, produzem novos sentidos e outras formas de pensar o trabalho, avançando em muitos aspectos nas proposições da Política Nacional de Saúde Bucal.

Um dos intuitos é estimular movimentos de integração entre temas ministrados nas disciplinas dos semestres iniciais dos cursos de odontologia com os estágios curriculares supervisionados realizados nos semestres finais. Compreende-se o projeto terapêutico singular como um "conjunto de propostas e de condutas terapêuticas articuladas, para um sujeito individual ou coletivo, resultado da discussão coletiva”. Didaticamente os seguintes momentos são vivenciados:

- Diagnóstico: avaliação das condições de vida e de saúde e doença dos indivíduos possibilitando a identificação da sua situação de vulnerabilidade. Procurando sempre captar se contexto: o trabalho, a cultura, a família e a rede social.

- Definição de propostas e metas: construção de propostas de curto, médio e longo prazo, e negociação com o indivíduo. Definição de tarefas e responsabilidade.

- Reavaliação: acompanhamento e discussão sobre a evolução da situação e as devidas correções de rota. $^{7}$

A criação dos projetos terapêuticos singulares em saúde bucal obedece aos princípios e diretrizes do processo de trabalho em saúde bucal contidos na política nacional de saúde bucal, assim como, as carac- terísticas da organização da atenção da saúde bucal segundo os ciclos de vida. ${ }^{5}$

\section{DESAFIOS DOS ESTÁGIOS NO SUS}

Se o estágio pautar-se pelo senso comum de "na prática a teoria é outra”, de lócus privilegiado de aprendizado ele se transforma em lócus privilegiado da construção esquizofrênica do cuidador. $O$ mundo da vida, das relações, das representações, dos afetos, das hierarquias, o sistema de signos, símbolos, a historicidade da instituição, necessariamente, a definem enquanto local de estágio. A circulação da palavra, o grau de democracia, o tipo de gestão, inclusive a gestão do sofrimento no trabalho são questões que deverão ser discutidas. As redes de subjetividades, a autodeterminação, as podencialidades e os limites da instituição, dos sujeitos e atores sociais. A presença e o manejo dos conflitos laborais. Deve-se ter a noção que a instituição é o lugar de movimento e não de cristalização. O campo de estágio deve propiciar a vivência da alteridade enquanto questão sócio-política, portanto, potencializadora do humano.

Considerando que as redes de atenção e ensino em saúde bucal ainda encontram-se em processo de estruturação, as experiências de estágios no SUS vivenciadas pela $\mathrm{FO} /$ UFRGS apresentam desafios a serem superados. Alguns deles podem ser assim delineados:

- a expansão limitada da APS e, consequentemente, dos campos de estágios;

- a necessidade de avanços nas discussões sobre o papel, atribuições e institucionalizações do preceptor/trabalhador e do tutor/docente;

- as incompreensões, ainda persistentes, a respeito dos estágios, tanto na IES como na gestão e nos serviços do SUS;

- as questões de financiamento;

- o discurso hegemônico da clínica liberal-privatista e seus reflexos num embate constante entre tutores/preceptores e discentes;

- os limites impostos pelo desenho fragmentado da rede de atenção em saúde.

A integração do ensino no serviço é eixo fundamental dos processos de mudanças que vem ocorrendo tanto no ensino superior como nos modelos e práticas públicos. Porém para que isso seja possível torna-se necessário a análise e divulgação de como essas experiências vêm sendo desenvolvidas, seus embates e suas conquistas. 


\section{ABSTRACT}

\section{Curriculum internships in SUS: experiences of the School of Dentistry of UFRGS}

One of the main objectives of the proposed paradigmatic shift in dental education in the country has been to shape the professional profile of the dentist in order to make it more suited to the needs dictated by the Brazilian Public Health System (SUS). However, this dental education model is still being shaped and there is no consensus in respect to how these professionals should be trained. The aim of this article was to describe and evaluate the pedagogical, technical and policy processes created over the course of implementing the new curricular dental internships at the School of Dentistry of the Federal University of Rio Grande do Sul. The paper also discusses the aspects involved in redirecting dental professional training towards SUS operations. Written and oral works of the teaching staff, documents and institutional reports, as well as school research were all analyzed. Professional education / health service integration is the fundamental axis of the changes in the processes taking place both in higher education and public health service actions. However, in order for such processes to take place in effect, it is necessary to analyze and disseminate how these experiences have developed, examining the struggles and the achievements encountered along the way.

\section{DESCRIPTORS}

Education. Training. Primary health. Oral health. Community outreach. "

\section{REFERÊNCIAS}

1. Antunes, R (org.). Riqueza e miséria do trabalho no Brasil. São Paulo: Editora Boitempo. Coleção Mundo do Trabalho. 2006.

2. Bercht, S. Contribuições para o estágio extra-muros da Faculdade de Odontologia da Universidade Federal do Rio Grande do Sul. Texto mimeografado. Departamento de Odontologia Preventiva e Social. Faculdade de Odontologia Universidade Federal do Rio Grande do Sul. Porto Alegre. 2008.

3. Brasil. Ministério da Saúde. Lei $n^{\circ} 8.080$, de 19 de setembro de 1990. Dispõe sobre as condições para a promoção, proteção e recuperação da saúde, a organização e o funcionamento dos serviços correspondentes e dá outras providencias. Diário Oficial [da] República Federativa do Brasil, Brasília; 20 set. 1990. Disponível em: < http://www.planalto.gov.br/ccivil_03/Leis/ L8080.htm > . Acesso em: 1 nov. 2010.

4. Brasil. Ministério da Educação e do Desporto CNE. Resolução CNE/CES 3/2002. Diário Oficial da União, Brasília, 4 de mar- ço de 2002. Seção 1, p. 10. Disponível em: < http:/ / portal.mec. gov.br/cne/arquivos/pdf/CES032002.pdf > . Acesso em: 1 nov. 2010.

5. Brasil. Ministério da Saúde. Secretaria de Atenção à Saúde. Departamento de Atenção Básica. Coordenação Nacional de Saúde Bucal. Diretrizes da Política Nacional de Saúde Bucal. Brasília; 2004. Disponível em: < http://bvsms.saude.gov.br/ bvs/publicacoes/politica_nacional_brasil_sorridente.pdf > . Acesso em: 1 nov. de 2010.

6. Brasil. Ministério da Saúde. Conselho Nacional de Saúde. Relatório Final da III Conferência Nacional de Saúde Bucal. Brasília: Ministério da Saúde; 2005. Disponível em: < http:/ / conselho.saude.gov.br/biblioteca/Relatorios/saude_bucal. pdf $>$. Acesso em: 1 nov. 2010.

7. Brasil. Ministério da Saúde Secretaria de Atenção à Saúde Núcleo Técnico da Política Nacional de Humanização. Clínica ampliada, equipe de referência e Projeto terapêutico singular. 2. ${ }^{\text {a }}$ ed. Série B. Textos Básicos de Saúde Brasília - DF; 2007.

8. Brasil. Ministério da Saúde. Ministério da Educação. Programa Nacional de Reorientação da Formação Profissional em Saúde - Pró-Saúde: objetivos, implementação e desenvolvimento potencial. Brasília: Ministério da Saúde; 2007. Disponível em: < http://www.prosaude.org > . Acesso 5 jan. 2011.

9. Brasil. Ministério da Saúde. Secretaria de Gestão do Trabalho e da Educação na Saúde. Programa de Educação pelo Trabalho para a Saúde - PET-SAÚDE. 2009a. Disponível em: < http:// www.prosaude.org/not/prosaude-maio2009/resumoPETSAUDE-29-04-09.pdf > . Acesso em: 2 jun. 2010.

10. Brasil. Ministério da Saúde. Portaria/GM/MS nº1.966, de 20 de agosto de 2007. Dispõe sobre as diretrizes para a implementação da Política Nacional de Educação Permanente em Saúde e dá outras providências. Diário Oficial da República Federativa do Brasil, Brasília; 2007.

11. Campos GWS. Clínica e saúde coletiva compartilhadas: teoria Paidéia e reformulação ampliada do trabalho em saúde. In: Campos GWS, Minayo MCS, Akerman M, Júnior MD,Carvalho YM. Tratado de Saúde Coletiva. 2. ed. São Paulo: Hucitec, 2008. Cap. 2, p. 41-80.

12. Ceccim RB. Onde se lê Recursos Humanos da Saúde, leia-se Coletivos Organizados de Produção da Saúde: Desafios Para a Educação. In: Pinheiro R, Mattos RA. (Orgs.) Construção Social da Demanda. Rio de Janeiro: CEPESC/UERJ: ABRASCO, 2005. p.161-80.

13. Lampert JB. Avaliação do processo de mudança na formação médica. In: Marins JJN. e outros (Orgs.). Educação médica em transformação: instrumentos para a construção de novas realidades. São Paulo: Hucitec, 2004. p.245-66.

14. Lemos VMA. Disciplina Estágio de Saúde Pública II. Apresentação Oral Oficina ABCD. Porto Alegre; 2009.

15. Lemos VMA, Nunes AA, Rosa RA, Rossoni E, Slavutzky SMB, Toassi RFC, Warmling M. Integração ensino-serviço-comuni- 
Estágios curriculares no SUS: experiências da Faculdade de Odontologia da UFRGS • Warmling CM, Rossoni E, Hugo FN, Toassi RFC, Lemos VA, Slavutzki SMB, Bercht S, Nunes AA, Rosa AR

dade no estágio da atenção básica da FOUFRGS: desafios e perspectivas. In. ABENO. Anais $45^{\mathrm{a}}$ Reunião da Associação Brasileira de Ensino Odontológico. Disponível em: < http:// www.abeno.org.br/index.php?option =com content\&view=article\&id $=97$ : resumos-dos-trabalhosapresentados\&catid= 42:avisos > . Acesso em: 20 jan. 2011.

16. Morin E. A cabeça bem-feita: repensar a reforma, reformar o pensamento. Trad.JACOBINA, E. 8 ed. Riode Janeiro:Bertrand Brasil; 2003.

17. Narvai PC. Saúde bucal coletiva: um conceito. Odontologia e Sociedade, São Paulo. 2001, 3 (1/2): 47-52.

18. Toassi RFC. O embate do processo de implantação de um currículo modular na Educação Superior: o curso de Odontologia da UNIPLAC, Lages - SC. 2008. 188 f. Tese (Doutorado em Educação) - Faculdade de Educação, Pontifícia Universidade Católica do Rio Grande do Sul, Porto Alegre; 2008.

19. Toassi RFC et al.. Integração ensino-serviço-comunidade: o estágio na atenção básica à saúde na graduação em odontologia. Apresentação Oral. Salão de Ensino UFRGS. Porto Alegre; 2010.

20. Universidade Federal do Rio Grande do Sul. Faculdade de Odontologia. Projeto Político Pedagógico. Porto Alegre; 2005. Disponível em < http//www.ufrgs.br/odonto/projeto_político.pdf $>$. Acesso em 16 jun. 2011.

21. Universidade Federal do Rio Grande do Sul. Faculdade de Odontologia. I Carta Acordo estabelecida entre a Fundação de Apoio da Universidade Federal do Rio Grande do Sul (FAURGS) e a Organização Pan-Americana da Saúde/Organização Mundial da Saúde (OPAS/OMS). Porto Alegre; 2007. Disponível em: < http:/ /www.ufrgs.br/odonto > . Acesso em: 5 jun. 2011.

22. Universidade Federal do Rio Grande do Sul. Faculdade de Odontologia. II Carta Acordo estabelecida entre a Fundação de Apoio da Universidade Federal do Rio Grande do Sul (FAURGS) e a Organização Pan-Americana da Saúde/Organização Mundial da Saúde (OPAS/OMS). Porto Alegre; 2009.
Disponível em: http://www.ufrgs.br/odonto/ Acesso em junho de 2011.

23. Universidade Federal do Rio Grande do Sul Faculdade de Odontologia. Resumo do pró-saúde faculdade de odontologia da Ufrgs. 2009. Disponível em: http://www.prosaude.org/ odo/resumo/UFRGS_ODO.pdf Acesso em: 01 novembro de 2010.

24. Universidade Federal do Rio Grande do Sul. Faculdade de Odontologia. Departamento de Odontologia Social e Preventiva. Estágio Curricular Supervisionado I da Odontologia. Plano de Ensino Estágio Curricular Supervisionado I da Odontologia 2010/01. Porto Alegre; 2010a.

25. Universidade Federal do Rio Grande do Sul. Faculdade de Odontologia. Departamento de Odontologia Social e Preventiva. Estágio Curricular Supervisionado I da Odontologia. Resultados avaliação preceptores 2010.01. Porto Alegre; 2010.

26. Universidade Federal do Rio Grande do Sul. Faculdade de Odontologia. Relatórios Oficina de Integração ensino e serviço 2010. Porto Alegre; 2010. Disponível: < http://www.ufrgs. br/odonto/ > . Acesso em: 10 mar. 2011.

27. Universidade Federal do Rio Grande do Sul. Faculdade de Odontologia. Departamento de Odontologia Social e Preventiva. Estágio Curricular Supervisionado II da Odontologia. Plano de Ensino Estágio Curricular Supervisionado II da Odontologia 2011/01. Porto Alegre; 2011.

28. Warmling CM. Da autonomia da boca: práticas curriculares e identidade profissional na emergência do ensino da odontologia brasileiro. Revista História Ciência e Saúde Manguinhos (no prelo) Rio de Janeiro; 2011.

29. Warmling CM, Lemos VMA, Rossoni E, Toassi RF, Slavutzsky SB, Rosa AR, Nunes AA. Educação permanente através da extensão universitária: oficinas e debates na FO/UFRGS. Texto mimeografado. Escola de Saúde Pública. Porto Alegre; 2010.

Recebido em 07/07/2011 Aceito em 25/07/2011 